\title{
Using Data-Driven Approach for Modeling Timing Parameters of American Sign Language
}

\author{
Sedeeq Al-khazraji \\ Rochester Institute of Technology (RIT) \\ Golisano College of Computing and Information Sciences \\ 20 Lomb Memorial Drive, Rochester, NY 14623 USA
}

sha6709@rit.edu

\begin{abstract}
While many organizations provide a website in multiple languages, few provide a sign-language version for deaf users, many of whom have lower written-language literacy. Rather than providing difficult-to-update videos of humans, a more practical solution would be for the organization to specify a script (representing the sequence of words) to generate a signlanguage animation. The challenge is we must select the accurate speed and timing of signs. In this work, focused on American Sign Language (ASL), motion-capture data recorded from humans is used to train machine learning models to calculate realistic timing for ASL animation movement, with an initial focus on inserting prosodic breaks (pauses), adjusting the pause durations for these pauses, and adjusting differentials signing rate for ASL animations based on the sentence syntax and other features. The methodology includes processing and cleaning data from an ASL corpus with motion-capture recordings, selecting features, and building machine learning models to predict where to insert pauses, length of pauses, and signing speed. The resulting models were evaluated using a cross-validation approach to train and test multiple models on various partitions of the dataset, to compare various learning algorithms and subsets of features. In addition, a user-based evaluation was conducted in which native ASL signers evaluated animations generated based on these models. This paper summarizes the motivations for this work, proposed solution, and the potential contribution of this work. This paper describes both completed work and some additional future research plans.
\end{abstract}

ACM Reference format:

Sedeeq Al-khazraji. 2018. Using Data-Driven Approach for Modeling Timing Parameters of American Sign Language. In Proceedings of the 2018 International Conference on Multimodal Interaction (ICMI'18), October 16-20, 2018, Boulder, CO, USA. ACM, New York, NY. 4pages. DOI: https://doi.org/10.1145/3242969.3264965

\section{KEYWORDS}

Accessibility for Deaf or Hard-of-Hearing; Machine Learning; Natural Language Processing; Human Computer Interaction.

Permission to make digital or hard copies of all or part of this work for personal or classroom use is granted without fee provided that copies are not made or distributed for profit or commercial advantage and that copies bear this notice and the full citation on the first page. Copyrights for components of this work owned by others than ACM must be honored. Abstracting with credit is permitted. To copy otherwise, or republish, to post on servers or to redistribute to lists, requires prior specific permission and/or a fee. Request permissions from Permissions@acm.org.

ICMI '18, October 16-20, 2018, Boulder, CO, USA

(C) 2018 Association for Computing Machinery.

ACM ISBN 978-1-4503-5692-3/18/10\$15.00 https://doi.org/10.1145/3242969.3264965

\section{INTRODUCTION}

American Sign Language (ASL) is used as a primary method of communication for more than 500,000 people in United States. ASL is a full natural language using movements of the hands, head, body, and face to convey the sentence. ASL a different language than English, it is not a simple representation of an English language sentence using movements; instead, ASL has its own syntax structure, word order, and lexicon which is different from spoken and written English.

While on one hand there are many Deaf or Hard-of-Hearing DHH individuals with very good English literacy, on the other hand, there are some $\mathrm{DHH}$ individuals who experienced a low level of language-exposure during their childhood and may have a lower level of English language literacy. As a matter of fact, standardized testing has shown that the majority of deaf secondary-school graduates in the United States have English reading level typical of U.S. students of age of ten. Because of the linguistic differences between the two languages (ASL \& English), there are many individuals who are fluent in American Sign Language but have many difficulties reading English text.

As a result of these literacy differences, some English text on websites may be too difficult to read for some individuals who are deaf. A simple solution to make websites more accessible is by adding videos of a human signer (who perform ASL signing) to websites, but this is not practical when information is updated frequently or generated automatically based on a query. A video would need to be re-recorded, and this process would be costly and time-consuming. An alternative solution would be to use computer animations carefully produced by a professional animation expert, but again this would be a time-consuming process. Accordingly, many researchers [7] [8] investigate building software that generates understandable ASL animations of a virtual human singer in a semi-automatic fashion from an easy-to-update script. The challenge is that this software must configure the animation so that the movements are accurate and easily understood by signers.

This paper explains my current Ph.D. research plans of using motion-capture data that our lab has previously recorded from human signers to build predictive models for Pause Insertion, adjusting Pause Duration, and adjusting Differential Signing Rate in ASL animations. (In subsequent work, I will investigate models of acceleration curves). Specifically, I am investigating building machine learning models for: 
- Pause Insertion: building a predictive model to decide where should we insert a prosodic break (a pause) within different words in ASL sentence.

- Pause Duration: If the above model decides to insert a pause, so what is the time-duration (or length) of this break/pause.

- Differential Signing Rate: building a machine learning model to select the best signing speed for each word within ASL sentences.

Previous studies at our lab [5] have shown that adding linguistically motivated pauses and adjusting signing rates will improve signers' understandability of ASL animation (as measured on a comprehension task); and tiny errors in these parameters will lead to less understandable animations. My ultimate goal is to automate this aspect of animation synthesis and to create understandable and realistic ASL animation with minimum human effort.

\section{RELATED LITERATURE}

There are many research areas related to this work:

1) Linguistic Research on Speed and Timing for ASL Signing: Prior psycholinguistic research has explored timing parameters in ASL and English based on video observations. These studies discovered some relations between timing parameters and the sentence syntactic structure [3], including proposals for some formulas for timing parameters based on sentence syntactic structure. The findings of this prior linguistic research played an important role in features selection stage for this work.

2) Rule-Based Research for ASL Animations: Prior researchers have made ASL animations by writing rules to control how to display an animated human to produce ASL with natural pauses and timing [5]. Huenerfauth [5] investigated the significance of pausing and speed in ASL animation; he built a model for the duration of the sign, the location of pauses, and the length of pauses in American Sign Language ASL. However, his model was based on rules that he wrote based on published findings in the psycholinguistics literature on ASL. He designed two algorithms, for Sign-Duration and for Pause-Insertion, to calculate sign duration time and to calculate pause location and length. The numerical constants in the rule-based approach were based on observations in prior linguistics literature about ASL, not on a machine-learning analysis of corpus data. This work will be referred to later as the "2008 Model" in Figures 2-3. Since ASL has complex linguistic interactions that influence how signs are produced, it is impractical to generate accurate ASL animations using a Rule-Based approach, to handle all cases that may arise.

3) Data-Driven Research: Most prior work on sign language has been rule-based (rather than data driven) because there are few available audio/video recordings that have been linguisticallyannotated. For "low resource" languages, many data-driven methods may not work (without special adaptations) because we do not have enough data. Since some small corpora for ASL have recently become available, we can now apply some data-driven approaches. There has been some preliminary research on generating ASL animations via a data-driven methods [11].
4) Speech Synthesis Research: Speech synthesis researchers generally use a part-of-speech of the words in a text to predict the prosodic breaks for English speech [10]. However, since reliable part-of-speech taggers do not exist for ASL, I investigate methods of predicting temporal parameters in ASL that: (1) do not required creating a part-of-speech tagger; and (2) required the smallest number of inputs (annotations).

\section{Research Questions}

In prior work, our lab has collected an ASL Motion-Capture Corpus [9], containing video and motion-capture recordings of fluent ASL signers, with linguistic annotation by experts of the words and linguistic structure of the signing. Our lab has previously used this resource to investigate different aspects of ASL animation: inflecting verb movement, facial expression, and spatial reference point locations. Given the success of these prior projects at using motion-capture recordings to build models of how human ASL signers behave (and then using these models to produce animations), I therefore intend to use a similar method to investigate the following research questions:

- RQ1: Can we predict locations where human signers should insert pauses in ASL sentences, to out-perform a baseline model that insert pauses at the end of the sentences only or the prior state-of-the-art rule-based approach of [5]?

- RQ2: Can we predict the time duration should these pauses should have, to out-perform a baseline model that assigns uniform duration or the prior rule-based approach of [5]?

- RQ3: Can we predict the differential signing rate (the speed of individual sign in ASL) during ASL sentences, to outperform a baseline model that assigns uniform speed to all signs or the prior rule-based approach of [5]?

- $\quad$ RQ4: Do these predictive models of pause insertion, pauses length, and adjusting signing rate lead to animations of ASL that deaf users prefer, as reported in an interview study?

- RQ5: Rather than considering a simple speed-multipler model for differential signing speed, would more complex models of signing speed (based on variations in acceleration curves during hand movements) out-perform our earlier models based on uniform models of differential signing rate?

This paper presents preliminary work on RQ1-RQ4, along with some discussion of future work plans to address RQ5.

\section{METHODOLOGY}

In this research, we use motion-capture data from a corpus of recordings of human ASL signers (which we had collected in prior work), to build predictive models for where to insert pauses, what duration the pauses should have, and what speed each sign should be performed at in an ASL sentence. We utilize the standard machine-learning methodological paradigm of preparing data, building a model, and evaluating its accuracy [4]. My tentative research plan is summarized as follows: 
- Data Preparation Process: I needed to process and extract relevant information from our lab's Motion-Capture Corpus, which contains motion-capture and linguistic annotation.

- Feature Engineering: I had to invent linguistic features that may be useful for modeling some linguistic phenomena and writing code to extract these features from raw corpus data. My goal is to prefer models based on a minimal set of features, so as to require as little input information as possible to the models (to reduce the manual human effort and time which is very costly), while maintaining a robust model.

- Building Machine Learning Models: The next phase was to build a predictive model using machine learning techniques. I trained different models to predict pause insertion, pause duration, and sign duration in ASL animation. For Pause Insertion model, I am using classification approach. While for Pause Duration and Differential Signing Rate, I am using regression machine learning modeling, as discussed in [1].

- Cross-Validation Model Evaluation: Due to scarce data, I use "cross-validation" training and testing, in which the dataset is separated into a number of equally sized groups called folds. The model is trained on a subset of the data (excluding onefold), and it is tested on this left-out fold. This process is repeated until all folds get a chance to be the left-out one. Accuracy is calculated by averaging across all folds.

- User-Based Evaluation of Animations: Since the goal of my research is to produce better animations of ASL for a deaf people who use ASL, there is value in conducting experimental studies of animation quality with these users. I am organizing files for ASL short stories (built using the preexisting, commercial Sign Smith Studio system), which could be used as stimuli for user-based evaluation studies. I modify the specification files to insert pauses or adjust other timing parameters, and then I regenerate the animations and show them to people who are deaf (and then ask participants for their subjective opinion). Our lab has published best-practices for how to conduct experiments with deaf users evaluating animations, which I follow [6].

\section{RESEARCH PLAN}

In this section, I provide a high-level overview of the research agenda for my work and identify what portion of the work has been completed and what is upcoming. Figure 1 shows the general representation for research agenda. The checked box represents completed work, and unchecked box represents upcoming work. Some activities are "(ready)" to begin soon.

As a summary of completed work in Pause Insertion modeling (Answering RQ1), I started with building a classification model to decide where to insert the pause in ASL animation, I have processed and extracted relevant information for three signers from our lab's Motion-Capture Corpus, which contains motion-capture and linguistic annotation. I identified the set of features and built a Linear-Chain CRF model to predict where to insert pauses between words in ASL sentences. My model has $80 \%$ accuracy which outperforms the proposed Baseline (Inserting pauses at the sentences boundaries). The details methodology of this work, more information about the selected features, and analyzing of different prediction approaches can be found on my published work in [2].

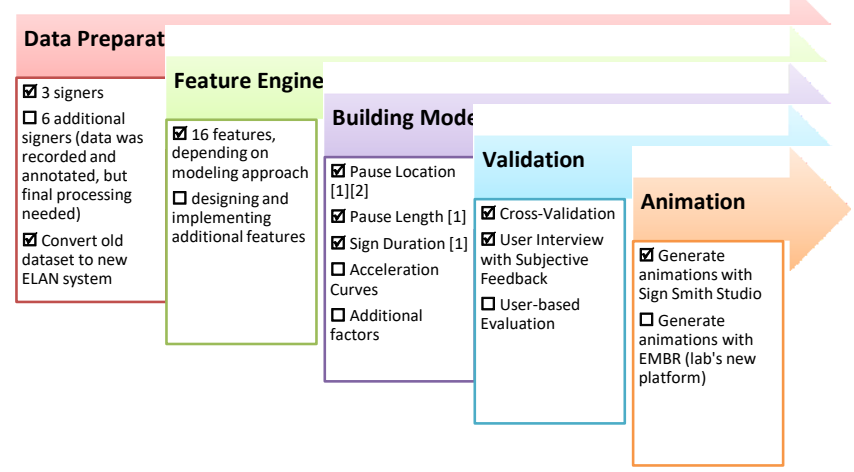

Figure 1: Overview of the Research Methodology.

My next completed work is described in a recent publication [1], which addressed RQ2-4, and presented models for Pause Insertion, Pause Duration, and Differential Signing Rate. Using our lab's Motion-Capture Corpus I generated a new dataset with expanded features. I built three models (Pause Insertion, Pause Duration, and Differential Signing Rate), each using a different feature subset. During modeling, I trained a Linear-Chain CRF model for Pause Insertion model. Next, I trained Gradient Boosting Regressor GBR to predict the Differential Signing Rate in ASL, and I used GridSearchCV to search the different training parameters, finding that using GBR with 50 estimators led to the best training model. Furthermore, I trained GBR with 100 estimators to predict the Pause Duration in ASL (here, GridSearchCV was used to search different training parameters). Finally, I evaluated my models by comparing each to a baseline, as follows: pauses inserted at the end of sentences only, an average (uniform) signing rate for all signs, and an average (uniform) duration for all pauses. Two forms of evaluation were conducted to compare my new models to these baselines:

- In a cross-validation study (discussed in [1], with results shown in Tables 1-3), the new models out-performed these baseline approaches. In addition, I compared my modeling approach with an earlier rule-based approach [5], which had previously been the state of the art. The new models also outperformed this prior state-of-the-art ASL timing model, as shown in Figures 2 and 3.

- I also conducted an interview study with DHH participants to learn their preferences about the animations generated with these models. I generated animations of ASL stories, and I conducted a user study where DHH participants saw different versions of animations of ASL stories, generated using the ASL-Speed model or the Baseline model. 75\% of participants preferred our new model. The details about modeling approach and the user study can be found at [1] 


\begin{tabular}{|l|c|}
\hline \multicolumn{1}{|c|}{ Classifier } & Accuracy (F1-Score) \\
\hline Linear-Chain CRF & $\mathbf{0 . 8 0}$ \\
\hline End of sentence Baseline & 0.77 \\
\hline Random locations Baseline & 0.64 \\
\hline
\end{tabular}

Table 1: Pause Prediction Model Results

\begin{tabular}{|l|c|}
\hline \multicolumn{1}{|c|}{ Regression Model } & RMSE \\
\hline Gradient Boosting Regressor & $\mathbf{0 . 4 5}$ \\
\hline Baseline & 0.50 \\
\hline
\end{tabular}

Table 2: Differential Signing Rate Prediction Model Results

\begin{tabular}{|l|c|}
\hline \multicolumn{1}{|c|}{ Regression Model } & RMSE \\
\hline Pause Duration (GBR) & $\mathbf{2 . 7 7}$ \\
\hline Baseline & 4.47 \\
\hline
\end{tabular}

Table 3: Pause Duration Prediction Model Results

One limitation of our current approach is that we treat the differential rate modifications of ASL signs as a multiplier; the actual speed adjustments may, in fact, consist of acceleration profiles which affect particular sub-portions of each sign, and we intend to explore such possibilities (RQ5) in future work. Also, I am exploring additional datasets for example, ASLLRP ${ }^{1}$ corpus.

\section{CONTRIBUTIONS}

My work contributes to generating realistic ASL animation using machine learning. I am building models for the temporal parameters of ASL animation, including pausing and speed.

\section{ACKNOWLEGEMENTS}

This material is based upon work supported by the National Science Foundation under award 1462280. We acknowledge the research contributions of colleagues: Larwan Berke, Abraham Glasser, Sushant Kafle, Matt Huenerfauth, and Peter Yeung.

\section{REFERENCES}

[1] Sedeeq Al-khazraji, Larwan Berke, Sushant Kafle, Peter Yeung, and Matt Huenerfauth. 2018. Modeling the Speed and Timing of American Sign Language to Generate Realistic Animations. The 20th International ACM SIGACCESS Conference on Computers and Accessibility (ASSETS '18), Galway, Ireland.

[2] Sedeeq Al-khazraji, Sushant Kafle, and Matt Huenerfauth. 2018. Modeling and Predicting the Location of Pauses for the Generation of Animations of American Sign Language. In Proceedings of the 8th Workshop on the Representation and Processing of Sign Languages: Involving the Language Community, The 11th International Conference on Language Resources and Evaluation (LREC 2018), Miyazaki, Japan.

[3] Francois Grosjean, Lysiane Grosjean, and Harlan Lane. 1979. The Patterns of Silence: Performance Structures in Sentence Production. Cognitive Psychology 11, 58-81.

[4] Barbora Hladka and Martin Holub. 2015. A Gentle Introduction to Machine Learning for Natural Language Processing: How to Start in 16 Practical Steps. Language and Linguistics Compass 9, 2, 55-76.

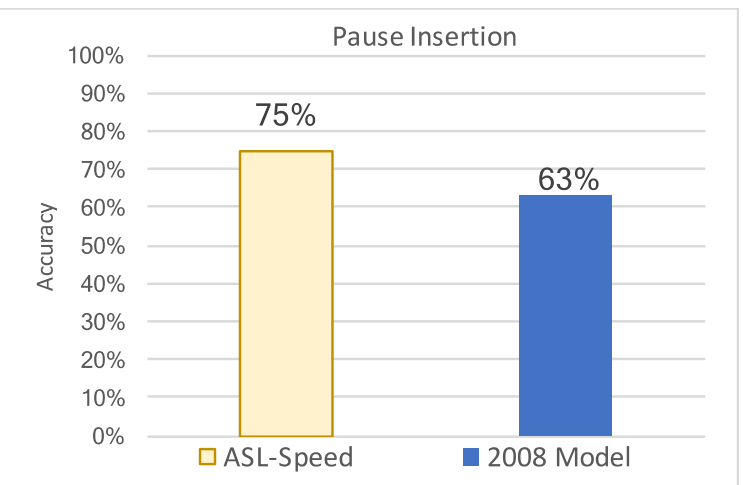

Figure 2: Comparing our new model (ASL-Speed) and a prior "2008 Model" from [5] on the Pause Insertion task.

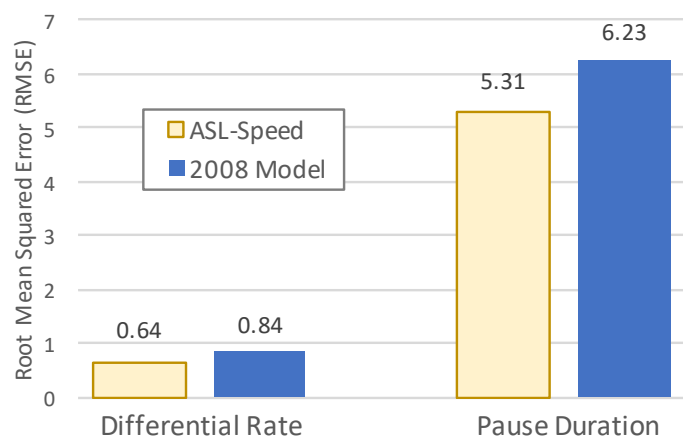

Figure 3: New model "ASL-Speed" out-performed the "2008 Model” [5] on Differential Rate and Pause Duration tasks.

[5] Matt Huenerfauth. 2008. Evaluation of a psycholinguistically motivated timing model for animations of American Sign Language. In Proceedings of the 10th international ACM SIGACCESS conference on Computers and accessibility (Assets '08). ACM, NY, USA, 129-136.

[6] Matt Huenerfauth and Hernisa Kacorri. 2015. Best Practices for Conducting Evaluations of Sign Language Animation. Jour. on Tech. and Persons with Disabilities 3, California State University, Northridge.

[7] Vince Jennings, Ralph Elliott, Richard Kennaway, and John Glauert. 2010. Requirements for a signing avatar. in Proc. Workshop on Corpora and Sign Language Technologies (CSLT), LREC.

[8] Kennaway, J. R., Glauert, J. R. W., and Zwitserlood, I. 2007. Providing Signed Content on the Internet by Synthesized Animation. ACM Trans. Comput.-Hum. Interact 14, 3, Article 15 (September 2007).

[9] Pengfei Lu and Matt Huenerfauth. 2012. CUNY American Sign Language Motion-Capture Corpus: First Release. Proceedings of the 5th Workshop on the Representation and Processing of Sign Languages: Interactions between Corpus and Lexicon, The 8th International Conf. on Language Resources and Evaluation (LREC 2012), Istanbul, Turkey.

[10] Alok Parlikar and Alan W. Black. 2012. Data-driven phrasing for speech synthesis in low-resource languages. in IEEE International Conference on Acoustics, Speech and Signal Processing (ICASSP), 4013-4016.

[11] Jérémie Segouat and Annelies Braffort. 2009. Toward the study of sign language coarticulation: methodology proposal. In Proc. of the Second Inter. Conf. on Advances in Comput.-Hum. Interac., 2009. 369-374.

\footnotetext{
${ }^{1}$ http://www.bu.edu/asllrp/
} 